\title{
Modellierung des Wärmeübergangs beim Blasensieden in freier Konvektion mit künstlichen neuronalen Netzen Teil II: Einstoffsysteme
}

\author{
Gerardo Diaz, Josef Schmadl, Thomas Schutt, Mihir Sen
}

\begin{abstract}
An artificial neural network (ANN) type multilayerperceptron with sigmoid activation function and errorbackpropagation learning algorithm was applied to predict heat transfer performances in a wide range of pressure and wall superheat at bubble boiling in free convection of two single component systems. For both systems the ANN prediction results were compared with the commonly used conventional correlation and gave in one case a comparable in the other a better accuracy. To improve the accuracy of ANN prediction it is possible to use interpolated experimental data for training and to optimise the network configuration.
\end{abstract}

\section{Einleitung}

Der Wärmeübergangskoeffizient (WÜK) $\alpha$, die Schlüsselgröße zur Berechnung wärmeübertragender Apparate, ist das Verhältnis zwischen Wärmestromdichte $\dot{q}\left[\mathrm{~W} / \mathrm{m}^{2}\right]$ und Wandüberhitzung DT [K] gemäß Gleichung

$\alpha=\frac{\dot{q}}{\Delta T}$

Da es für $\alpha$ wegen der großen Zahl der Einflußgrößen keine einheitliche Berechnungstheorie gibt, behilft man sich meist entweder mit gemessenen Werten oder, falls nicht vorliegend, mit empirischen und halbempirischen Berechnungsgleichungen. Im Falle des hier zu besprechenden Blasensiedens in freier Konvektion liegt in der deutschen Fachliteratur eine große Zahl zuverlässiger Messdaten sowohl für zahlreiche reine Stoffe als auch für - vor allem binäre - Stoffgemische vor. Für Berechnungen wird eine empirische Gleichung von der folgenden Form verwendet [1]:

$\frac{\alpha}{\alpha_{0}}=C_{W} \cdot F\left(p^{*}\right) \cdot\left(\frac{\dot{q}}{\dot{q}_{0}}\right)^{n\left(p^{*}\right)}$

mit

$C_{W}=\left(\frac{R_{a}}{R_{a 0}}\right)^{0,133} \cdot\left(\frac{\lambda \cdot \rho \cdot c}{\lambda_{0} \cdot \rho_{0} \cdot c_{0}}\right)^{0,25}$

und, eingeschränkt gültig für die meisten nichtwässrigen Medien,

$F\left(p^{*}\right)=1,2 \cdot p^{* 0,27}+\left(2,5+\frac{1}{1-p^{*}}\right) \cdot p^{*}$
$n=0,9-0,3 \cdot p^{* 0,3}$
Darin sind die Heizwandeigenschaften (Wandrauhigkeit, -geometrie und/oder Stoffdaten) in der Funktion $C_{W}$, die des Druckes ( $p$ oder $\mathrm{p}^{*}=\mathrm{p} / \mathrm{p}_{\mathrm{k}}$ ) in $\mathrm{F}\left(\mathrm{p}^{*}\right)$ und die der Wärmestromdichte im letzten Term der Gleichung, der außerdem die druckabhängige Steigung der $\log \alpha / \log \dot{q}$ Geraden enthält, zusammengefasst. Notwendig für die Berechnung ist, dass wenigstens ein Messwert $\alpha_{0}$ bei einem willkürlich wählbaren Bezugszustand (z. B. bei der Rauhigkeit $\mathrm{R}_{\mathrm{a} 0}=0,4 \mu \mathrm{m}$ einer Heizfläche aus Kupfer, dem normierten Druck $p_{0}^{*}=0,1$ und der Wärmestromdichte $\dot{q}_{0}=20 \mathrm{~kW} / \mathrm{m}^{2}$ ) bekannt ist, von dem aus dann auf andere, gewünschte Heizwände, Drücke oder Wärmestromdichten umgerechnet werden kann. Ist kein Messwert vorhanden, kann ein solcher mit der bekannten Stephan-Preusser-Gleichung anhand von Stoffdaten der siedenden Flüssigkeit berechnet werden [1, Ha]. Der Fehler ist dabei mit $\pm 15 \%$ relativ hoch.

Als Alternative zu der Berechnung des WÜK nach Gl. (2) bis (5) bieten sich KNN-Berechnungen an. Die in der Fachliteratur in genügender Zahl und Qualität vorliegenden experimentellen Daten dürften dabei als Trainingsmaterial geeignet sein. In dieser Arbeit werden erste Berechnungen von $\alpha$ für reine Stoffe mit einem künstlichen neuronalen Netz (KNN) im Vergleich zu der herkömmlichen Berechnungsmethode nach Gleichung (2) durchgefuihrt mit dem Ziel, vorab Möglichkeiten und Grenzen der KNNe für die Auslegung von Apparaten oder die Modellierung von Apparateleistungsgrößen auszuloten.

\section{Aufbau des verwendeten KNN und des zugehörigen Fortran-Programms}

Für die Berechnung des Wärmeübergangs beim Behältersieden steht ein voll verknotetes, in [2] ausfuihrlicher beschriebenes Multilayerperceptron zur Verfuigung mit I = 5 Schichten, $\mathrm{J}_{1}=2, \mathrm{~J}_{2}=7, \mathrm{~J}_{3}=5, \mathrm{~J}_{4}=3, \mathrm{~J}_{5}=1$ Neuronen jeweils pro Schicht (Abbildung 1).

Das Netz wird mit experimentellen Datensätzen $\left(\dot{q}, \Delta T, p^{*}\right)$ trainiert, wobei Wandüberhitzung und normierter Druck als Eingangsgrößen $\mathrm{X}_{0, \mathrm{j}}$ und die Wärmestromdichte in normierter Form als Vergleichswert $t_{l, j}$ für die Ausgangsgröße $y_{I, j}=y_{5,1}$ fungieren. Vor der Anwendung wird eine Trainings- und Testdatei erstellt. In ihr werden die Datensätze zeilenweise angeordnet und die einzelnen Eingangsgrößen spaltenweise. Dabei ist zu beachten, dass die Trainingsgrößen in den vorderen Spalten und die Vergleichsgröße für die Ausgangsgröße in der letzten Spalte geschrieben werden. Die 


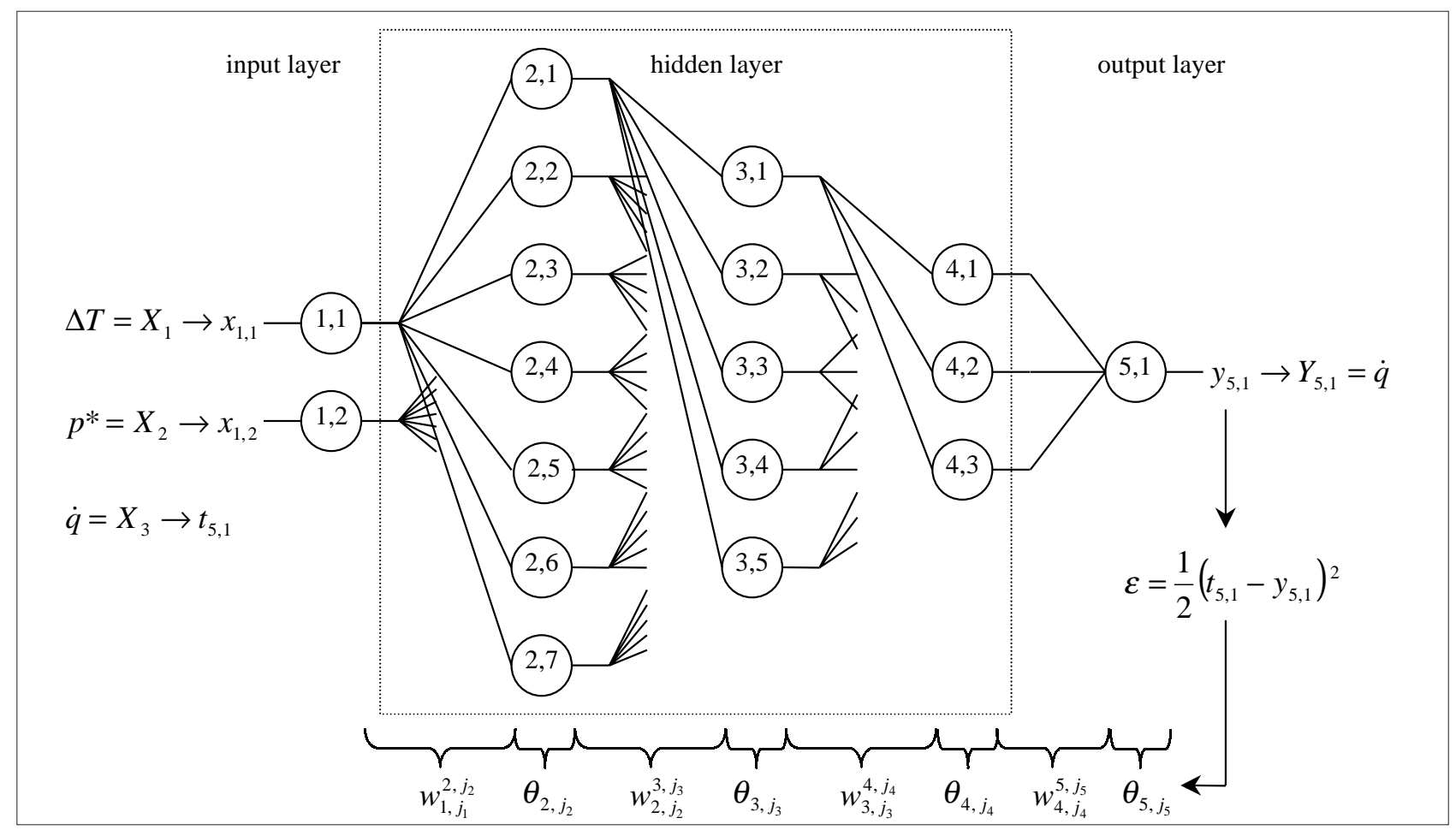

Abb. 1: Verwendetes Multilayerperceptron mit $I=5$ Schichten und $J_{i}$ Neuronen pro Schicht.

zeilenweisen Trainingssätze sollten möglichst nicht in Reihenfolge geordnet werden, um ein „Auswendiglernen“ zu Lasten der Interpolationsfähigkeit zu vermeiden. Über Gl. (1) lässt sich das Rechenergebnis wahlweise in der Form $\alpha=\alpha(\dot{q})$ oder $\dot{q}=\dot{q}(\Delta T)$ darstellen. Die Rauhigkeit wurde als Parameter nicht berüicksichtigt, da für die untersuchten Stoffe nicht ausreichend genaue Angaben zur Rauhigkeit vorliegen und diese im hier betrachteten Druckbereich ab $10 \%$ des kritischen Drukkes einen relativ geringen Einfluss ausüibt.

Die Eingangsdaten $X_{0, j}$ werden, wie üblich, für die erste Schicht normiert auf Werte $0<\mathrm{x}_{1, \mathrm{j}}<1$. Da aber ihre extremen Zahlenwerte sich um mehrere Zehnerpotenzen unterscheiden, kämen durch einfache Normierung Normierungswerte nahe 0 und 1 zustande, was die Empfindlichkeit der sigmoiden Aktivierungsfunktion stark beeinträchtigen würde. Deshalb wurden die Normierungsgrenzen eingeschränkt auf:

$0,15<x_{1, j}=\frac{\left(s_{\max }-s_{\min }\right) \cdot\left(X_{0, j}-X_{0, \min }\right)}{X_{0, \max }-X_{0, \min }}+s_{\min }<0,85$

mit $s_{\min }=0,15$ und $s_{\max }=0,85$.

Als Propagierungs- bzw. Aktivierungsfunktion dienen:

$x_{i, j}=\xi\left(y_{i-1, k}, w_{i-1, k}^{i, j}, \theta_{i, j}\right)=\theta_{i, j}+\sum_{k} w_{i-1, k}^{i, j} \cdot y_{i-1, k}$

$y_{i, j}=\Phi\left(x_{i, j}\right)=\frac{1}{1+e^{-k \cdot x_{i, j}}}$ für i $>1$

Gewichte und Bias-Werte wurden nach dem Errorbackpropagation-Algorithmus trainiert:

$$
\begin{aligned}
& w_{I-1, k}^{I, j}(m+1)=w_{I-1, k}^{I, j}(m)+\lambda \cdot\left(t_{I, j}-y_{I, j}\right) . \\
& y_{I, j} \cdot\left(1-y_{I, j}\right) \cdot y_{I-1, k} \text { für } \mathrm{i}=\mathrm{I}=5
\end{aligned}
$$

mit $\mathrm{j}=1$ und $1<\mathrm{m}<\mathrm{M}=10^{6}$ in dieser Arbeit.

$w_{i-1, k}^{i, j}(m+1)=w_{i-1, k}^{i, j}(m)+\lambda \cdot \delta_{i, j} \cdot y_{i-1, k}$

fuir $1<\mathrm{i}<\mathrm{I}=5$

$\theta_{i, j}(m+1)=\theta_{i, j}(m)+\lambda \cdot \delta_{i, j}$ für $1<i \leq I=5$

Das hier verwendete KNN-Beispiel ist in Fortran 90 programmiert und auf einer Unix-Workstation implementiert. Die Abbildungen 2-4 enthalten die wichtigsten Ablaufpläne. Es unterteilt sich in ein Hauptprogramm und 6 Unterprogramme. Hier sind lediglich das Hauptprogramm und die beiden wichtigsten Unterprogramme „subroutine training“ und „subroutine test“ dargestellt. In diesen Programmstrukturen werden folgende statische Variablen genutzt:

iconta Zählvariable für die Bildschirmausgabe der Zwischenergebnisse

icyc Zählvariable für die Iterationsschritte $1<\mathrm{m}<\mathrm{M}$

ncyclestot Anzahl der durchzufuihrenden Iterationen M ncycles Variable, die angibt, nach wie vielen Iterationen ein Zwischenergebnis an den Bildschirm ausgegeben werden soll

p Zeilennummer der Datensätze np Anzahl der vorhandenen Datensätze istat Variable zur Initialisierung (istat $=0$ ) der Gewichte und Bias-Werte im ersten Trainingszyklus 


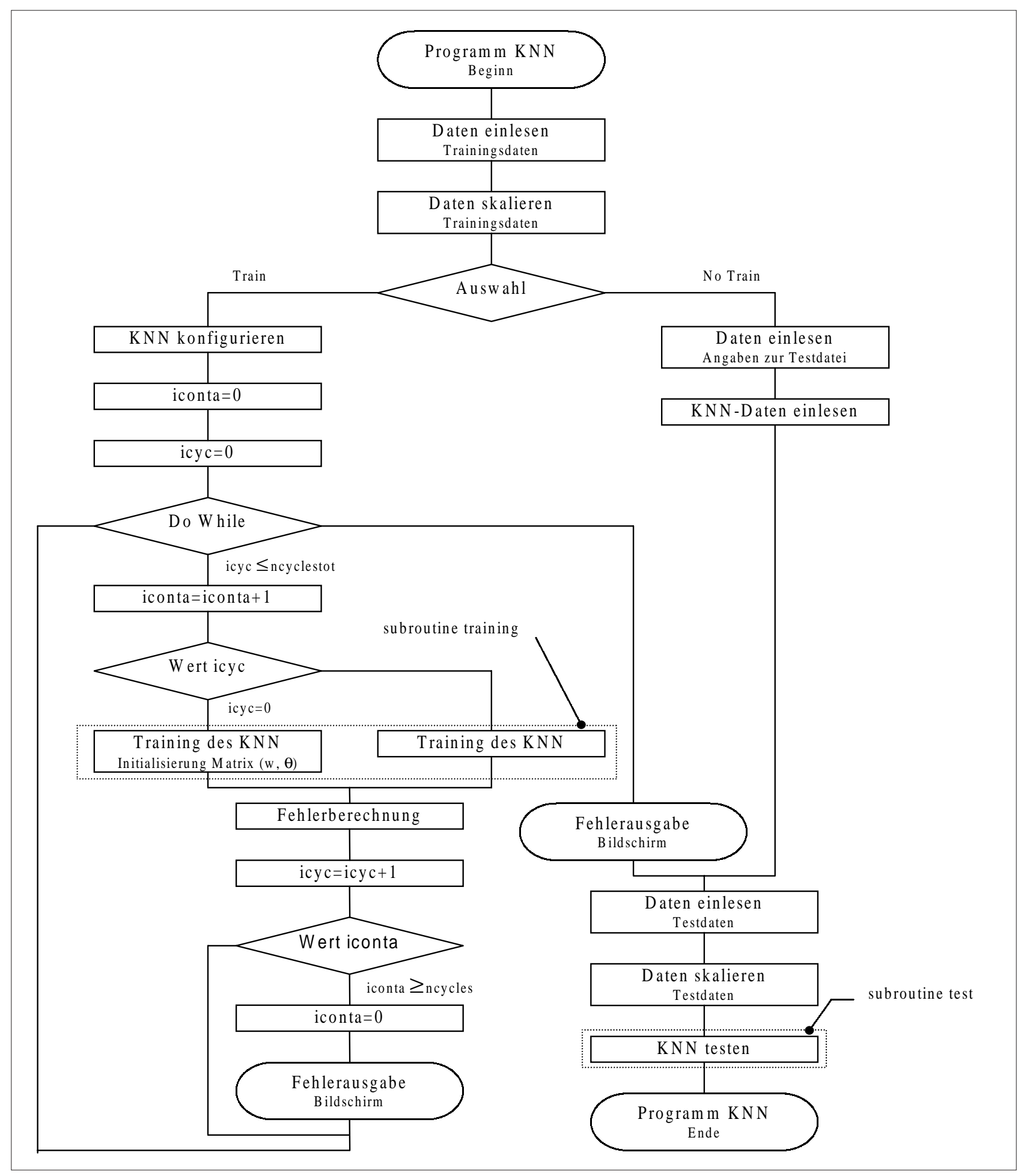

Abb. 2: Programmablaufplan Hauptprogramm

\section{Rechenergebnisse}

Das Netz wurde mit den von Schmadl [3] und Bayer [4] gemessenen Daten für die Stoffe Schwefelhexafluorid und Dichlordifluormethan jeweils getrennt im Bereich $0,5<\dot{q}<100 \mathrm{~kW} / \mathrm{m}^{2}$ und $0,100<\mathrm{p}^{*}<0,972$ trainiert, so dass stoffspezifische Gewichte und Bias-Werte ermittelt wurden. In der Testphase wurden für beide Stoffe interpolierte Messwerte bei den Wärmestromdichten $\dot{q}$ $=1,4,10,20,40 \mathrm{~kW} / \mathrm{m}^{2}$ und denselben Drücken, bei denen auch Messwerte vorliegen, verwendet. Vergleichsweise zu den KNN-Rechenwerten wurden bei denselben WSD- und Druckwerten VDI-Berechnungen nach Gl. (2) bis (5) mit $\alpha$ nach Gl. (1) durchgefuihrt. Da alle Messwerte mit elektrisch beheizten Kupferrohren ermittelt wurden und der Rauhigkeitseinfluss nicht berücksichtigt wurde, ist somit $\mathrm{C}_{\mathrm{W}}=1$ in $\mathrm{Gl}$. (2).

Zum Vergleich der KNN- und VDI-Rechenwerte wurden eine mittlere Abweichung $R$ und eine Standardabweichung $\sigma$ betrachtet für das Verhältnis zwischen gemessenem und berechnetem Wert $\mathrm{R}_{\mathrm{r}}[5]$ :

$R_{r}=\frac{\dot{q}_{\mathrm{exp}}}{\dot{q}_{\text {calc }}}$ 


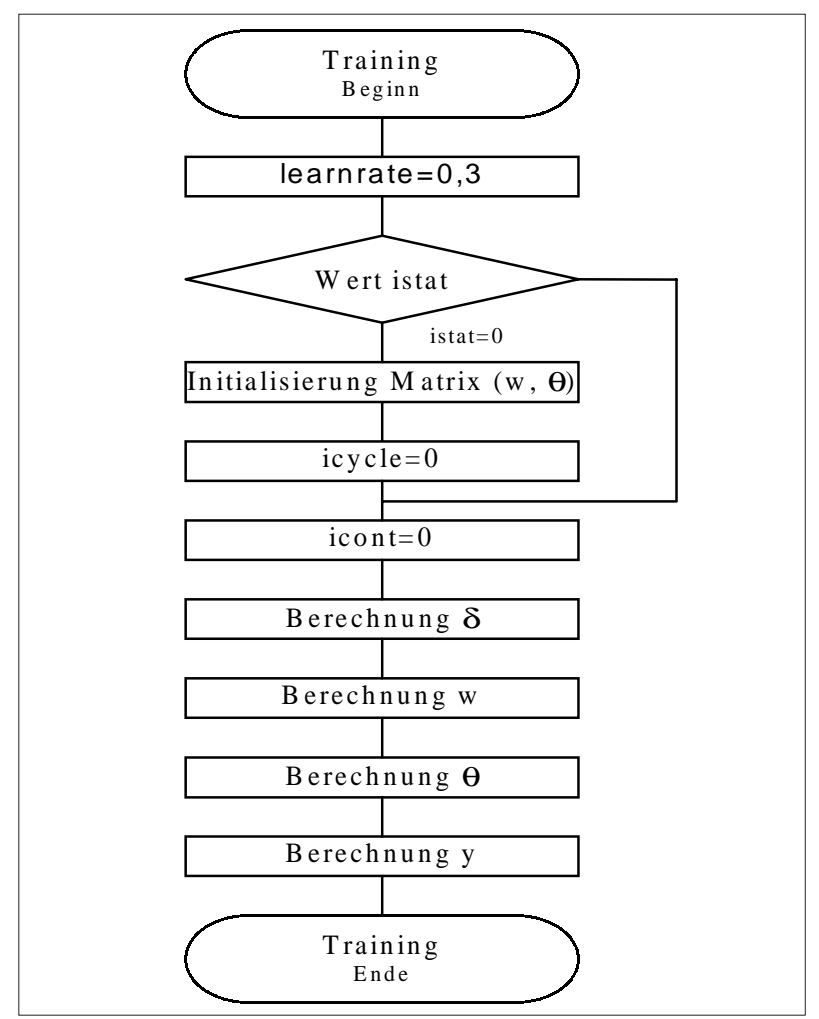

Abb. 3: Programmablaufplan „subroutine training“

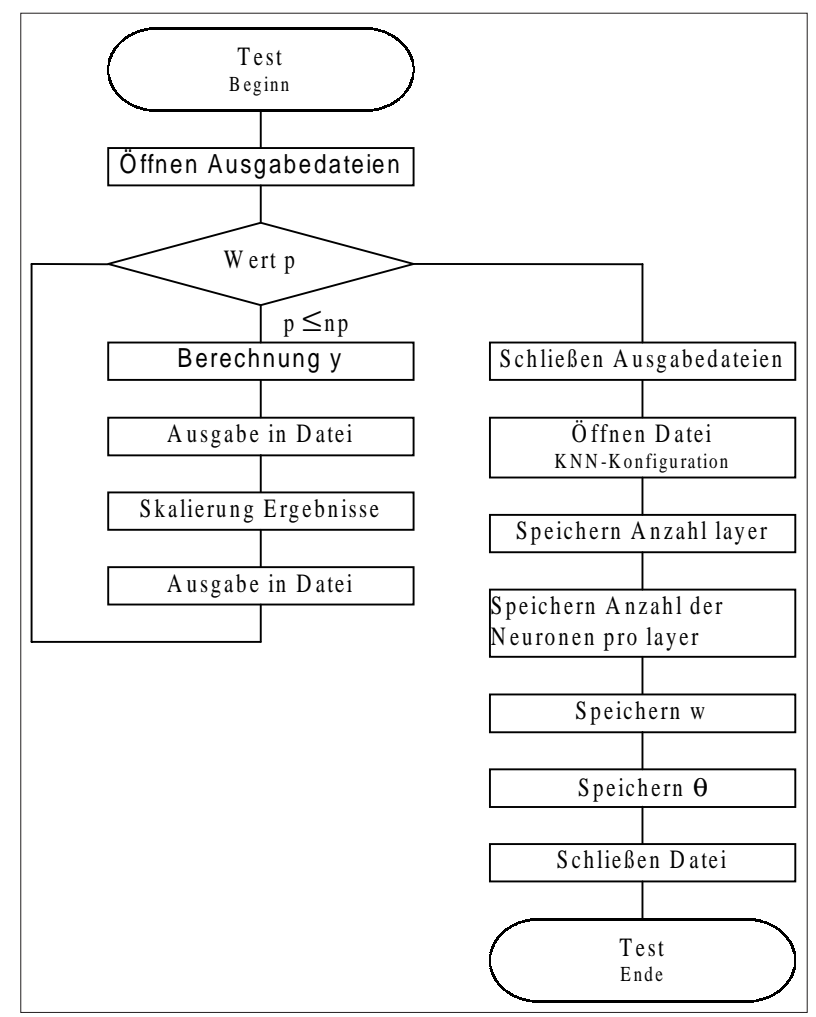

Abb. 4: Programmablaufplan „subroutine test“

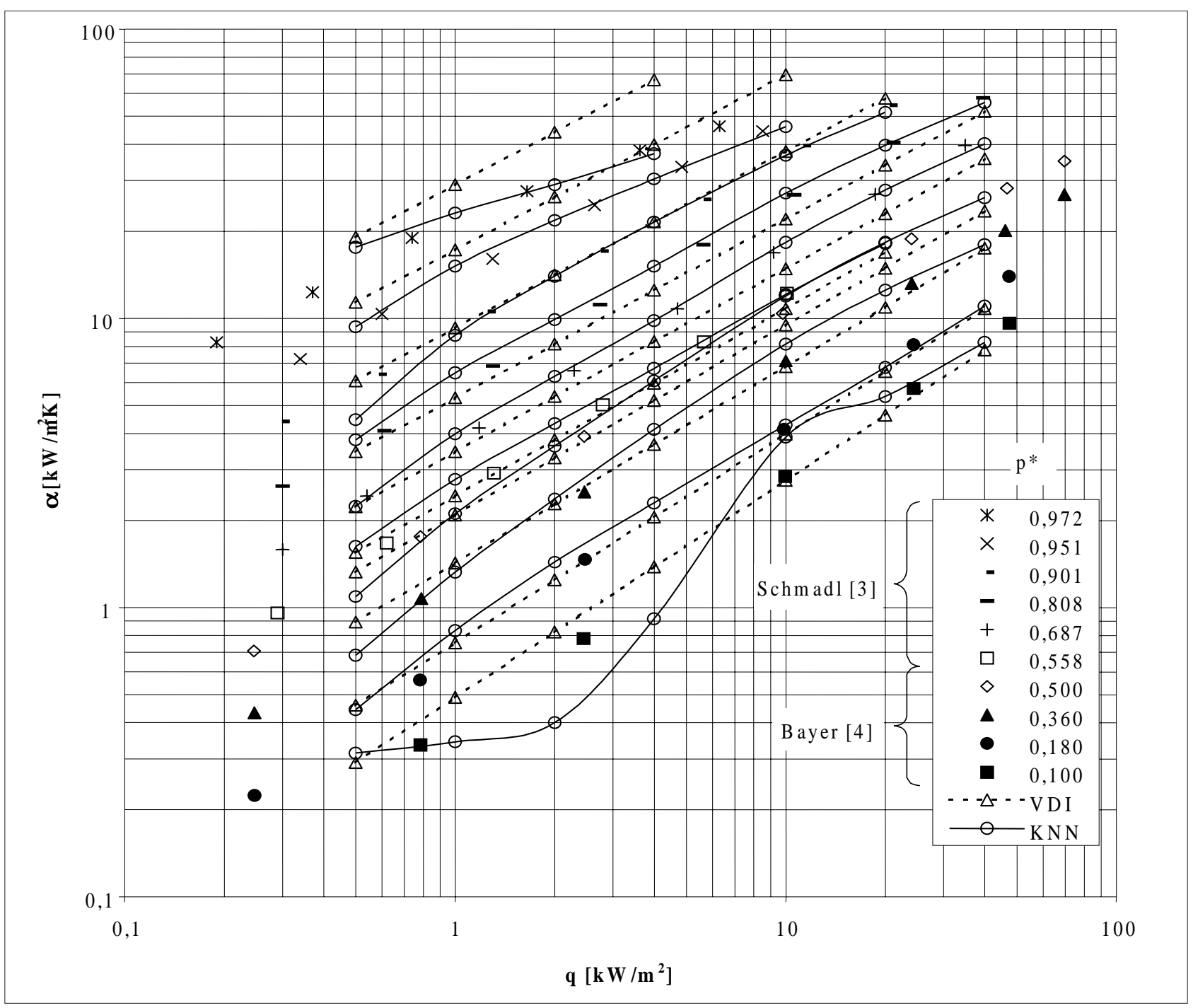

Abb. 5: Wärmeübergangskoeffizient $\alpha$ in Abhängigkeit von der Wärmestromdichte $\dot{q}$ bei verschiedenen normierten Siededrücken für SF ${ }_{6}$ Vergleich der Rechenergebnisse nach Gl. (2) (gestrichelte Linie) und der KNN-Rechenergebnisse (durchgezogene Linie) mit experimentellen Werten. 


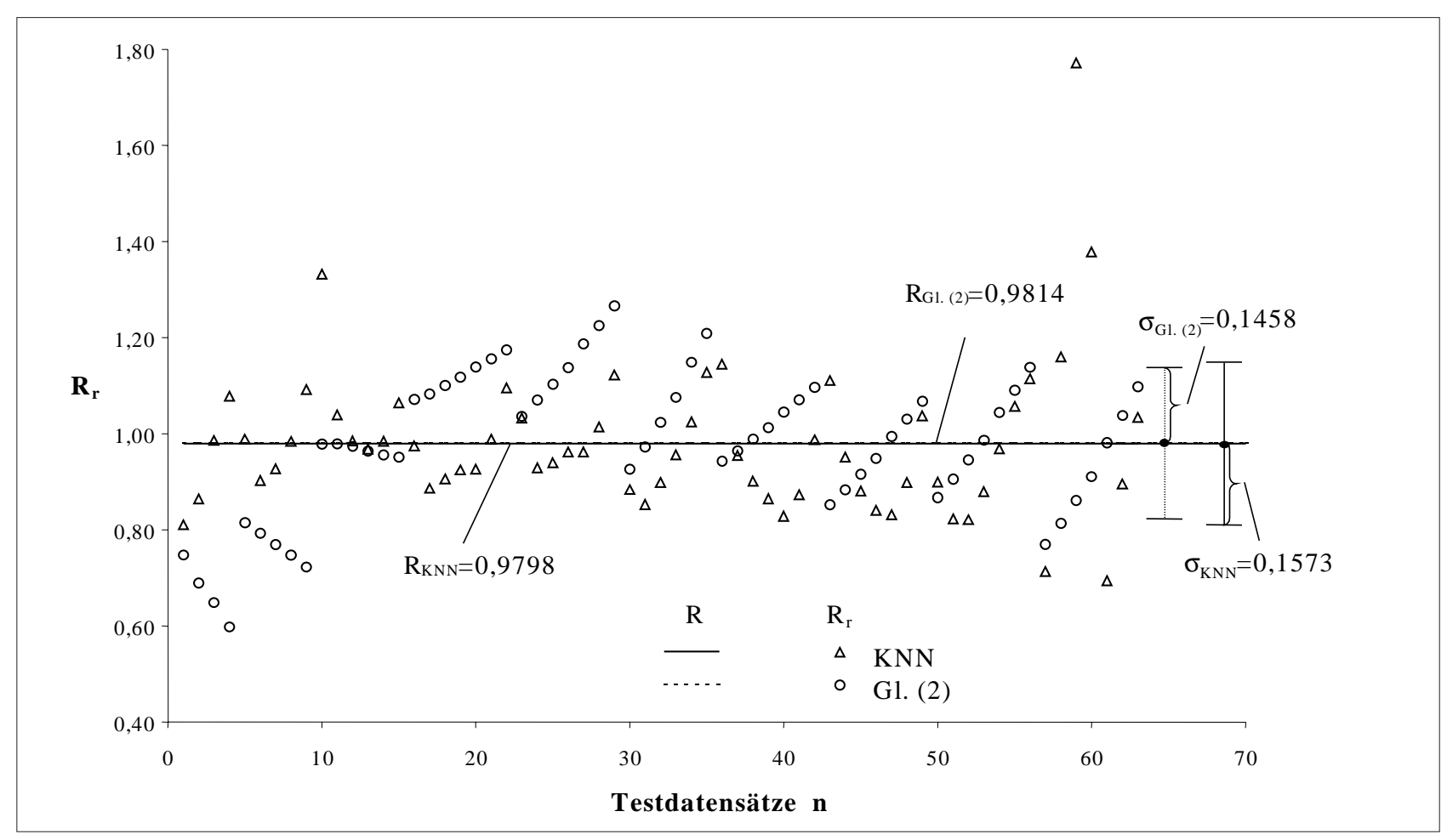

Abb. 6: Relative Abweichung $R_{r}$, mittlere Abweichung $R$ und Standardabweichung $\sigma$ für $S F_{6}$-Rechenwerte nach Gl. (2) und KNN.

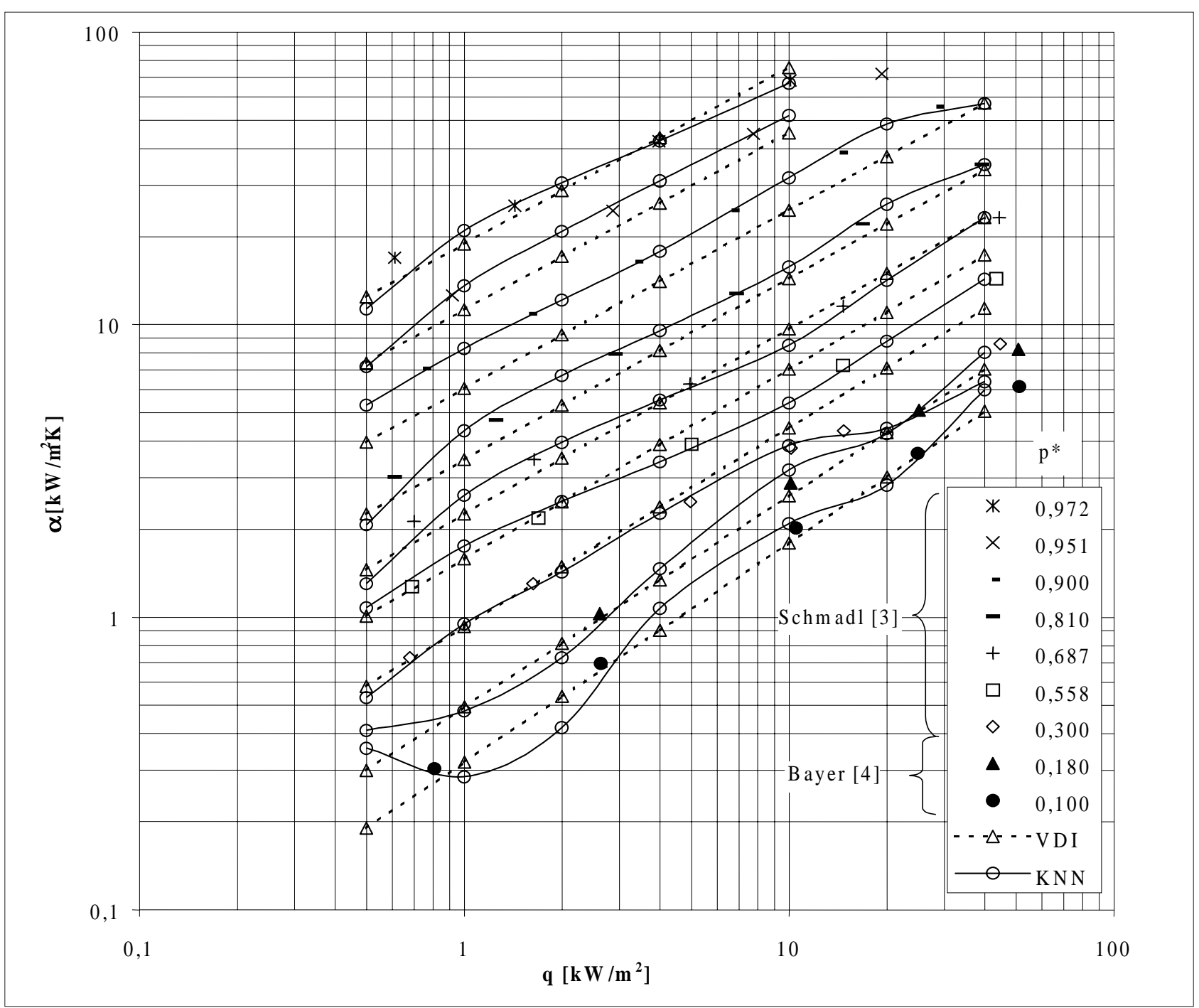

Abb. 7: Wärmeübergangskoeffizient $\alpha$ in Abhängigkeit von der Wärmestromdichte bei verschiedenen normierten Siededrücken für $\mathrm{CF}_{2} \mathrm{Cl}_{2}$. Vergleich der Rechenergebnisse nach Gl.(2) (gestrichelte Linien) und KNN (durchgezogene Linien) mit experimentellen Werten. 


$$
R=\frac{1}{N} \sum_{r=1}^{N} R_{r}
$$

$$
\sigma=\sqrt{\sum_{r=1}^{N} \frac{\left(R_{r}-R\right)^{2}}{N}}
$$

\section{1 $\mathrm{SF}_{6}$ - Ergebnisse}

In der doppeltlogarithmischen Darstellung des WÜK über der WSD in Abbildung 5 sind Rechenwerte nach Gl. (2) und KNN-Rechenwerte den zum KNN-Training verwendeten experimentellen Werten gegenübergestellt. Man sieht, dass größere Abweichungen zwischen Rechnung und Messung für die Rechenwerte nach Gl. (2) bei hohen Drücken und für die KNN-Rechenwerte beim niedrigen Druck auftreten. Bei mittleren Siededrücken sind beide Rechenmethoden vergleichbar gut.

In Abbildung 6 sind relative, mittlere und Standardabweichung, wie in Gl. (12) bis (14) definiert, dargestellt. Man sieht, dass die KNN-Berechnungen mit 0,9798 gegenüber 0,9814 eine nahezu gleich große mittlere Abweichung, aber mit 0,1573 gegenüber 0,1458 eine etwas größere Standardabweichung, d. h. eine größere Streuung aufweisen als die Berechnungen nach Gl. (2).

\section{2 $\mathrm{CF}_{2} \mathrm{Cl}_{2}$-Ergebnisse}

Die Berechnungen mit Difluordichlormethan ergaben ein ähnliches Ergebnis: Die Übereinstimmung mit den experimentellen Werten ist auch hier fuir Gl. (2) beim niedrigen normierten Druck besser als für das KNN, wie aus Abbildung 7 hervorgeht. Allerdings weist insgesamt das KNN für diesen Stoff mit 1,0262 gegenüiber 1,0678 einen geringeren mittleren Fehler $\mathrm{R}$ und mit 0,1423 gegenuiber 0,1455 eine geringere Standardabweichung auf als Gl. (2).

\section{Zusammenfassung}

In dieser Arbeit wurde die Leistungsfähigkeit eines KNN vom Typ Multilayerperceptron zur Vorausberechnung des Wärmeübergangskoeffizienten beim Blasensieden in freier Konvektion für zwei Einstoffsysteme untersucht. Im Vergleich zu der konventionellen Berechnungsgleichung (2) gibt das KNN die experimentellen Werte bei mittleren und hohen Drücken besser, bei niedrigen Drükken schlechter wieder. Die mittleren Abweichungen betragen beim Schwefelhexafluorid 2,0 \% für KNN und 1,9 \% fuir Gl. (2), beim Difluordichlormethan 2,6 \% bzw. 6,8 $\%$. Die Standardabweichungen sind nicht wesentlich verschieden und bewegen sich zwischen 0,14 und 0,16 . Verbesserungen in der Genauigkeit der KNN-Berechnungen lassen sich möglicherweise erzielen durch Training mit interpolativ geglätteten Messdaten und durch Optimierung der Netzkonfiguration.

\section{Verwendete Symbole und Abkürzungen in Formeln:}

$\mathrm{i}, 1$

Laufzahl der Schichten

$1<\mathrm{i}<\mathrm{I}, 1<\mathrm{I}<\mathrm{L}$

$\mathrm{j}, \mathrm{k}$

Laufzahl der formalen Neuronen im Layer i, $1<\mathrm{j}<\mathrm{J}_{\mathrm{i}}$, bzw. $1,1<\mathrm{k}<\mathrm{K}_{\mathrm{l}}$

m

Laufzahl der Iterationsschritte

$1<\mathrm{m}<\mathrm{M}$

$\begin{array}{ll}x_{i, j}, y_{i, j} & \text { Ein- und Ausgangswert des Neurons } \\ \mathrm{j} \text { in der Schicht } \mathrm{i}\end{array}$

$\theta_{i, j} \quad$ Bias-Wert des Neurons $\mathrm{j}$

in der Schicht $\mathrm{i}$

$w_{i-1, k}^{i, j}$

Gewicht der Synapse

(i-1, k) à (i, j)

$\xi \quad$ Propagierungsfunktion

$\Phi \quad$ Aktivierungsfunktion

$1 \quad$ Lernrate

$t_{I, j}=t_{5,1}=\dot{q} \begin{aligned} & \text { Trainingswert, hier: } \\ & \text { Wärmestromdichte }\end{aligned}$

n Laufzahl der Testdatensätze, $1 \leq n \leq N$

\section{Literatur}

[1] VDI-Wärmeatlas, Springer-Verlag, 8. Auflage, 1997

[2] Diaz, G.; Sen, M.; Schutt, T.; Schmadl, J.: Modellierung des Wärmeübergangs beim Blasensieden in freier Konvektion mit künstlichen neuronalen Netzen. Teil I: Literaturübersicht. Wissenschaftliche Beiträge TFH Wildau, Heft $2 / 2000$

[3] Schmadl, J.: Wärmeübergang beim Blasensieden binärer Stoffgemische unter hohem Druck. Dissertation Universität Karlsruhe 1982

[4] Bayer, A.: Untersuchungen zum Blasensieden von binären Stoffgemischen in einem großen Druckbereich. Dissertation Universität Karlsruhe, 1988

[5] Yang, K. T.; Sen, M. et al: Applications of Artificial Neural Networks and Genetics Algorithms in Thermal Engineering. In: CRC Handbook of Thermal Engineering, Section $4,620-661$

\section{Autoren}

\section{Gerardo Diaz}

Prof. Dr. Mihir Sen

University of Notre Dame

Department of Aerospace and Mechanical Engineering Notre Dame, IN 46556, USA

E-Mail: Mihir.Sen.1@nd.edu

Prof. Dr.-Ing. Josef Schmadl

Thomas Schutt

Technische Fachhochschule Wildau

Technikum für Thermische Verfahrenstechnik

E-Mail: jschmadl@igw.tfh-wildau.de 


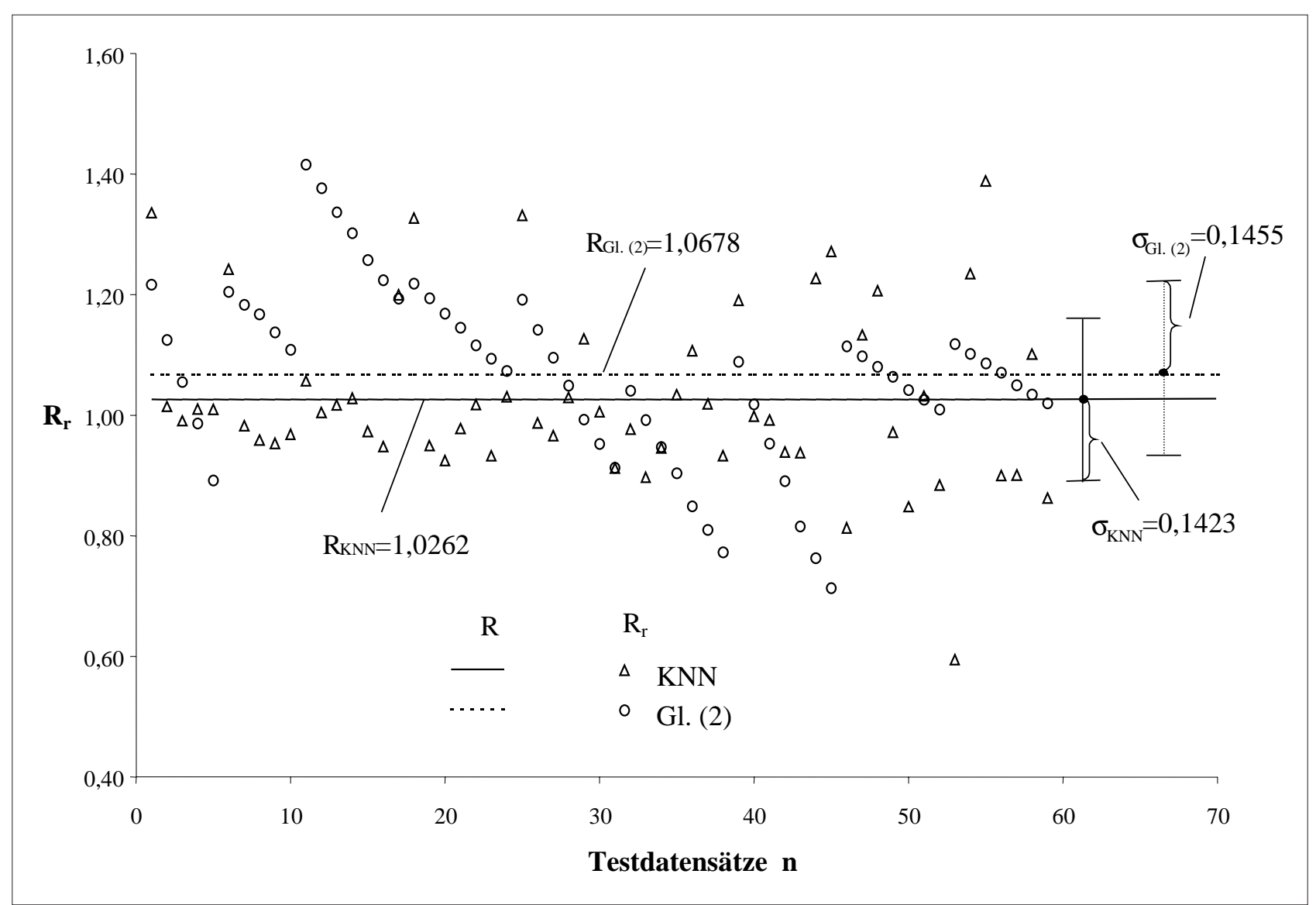

Abb. 8: Relative Abweichung $R_{r}$, mittlere Abweichung $R$ und Standardabweichung $\sigma$ für $\mathrm{CF}_{2} \mathrm{Cl}_{2}$-Rechenwerte nach Gl. (2) und KNN. 